\title{
AN EXPERIMENTAL LAB TO ENHANCE UNDERGRADUATE ELECTROMAGNETICS EDUCATION
}

\author{
David Garrett and Elise Fear \\ Department of Electrical and Computer Engineering, Schulich School of Engineering, University of Calgary \\ fear@ucalgary.ca
}

Electromagnetic field theory is a required part of the undergraduate electrical engineering curriculum. This material is taught in either one or two semester courses, encompassing concepts related to static and time-varying fields. These classes often focus on Maxwell's equations and application of mathematical tools required for problem solving. Simulation and visualization tools are often used to enhance student understanding of field distributions and wave propagation. However, hands-on experiences have the potential to deepen understanding and emphasize connections between theory and real-world applications. As RF and microwave equipment is expensive, it is difficult to sustainably outfit laboratories for large undergraduate classes. In this paper, we describe a lowcost, hands-on experimental lab that involves students designing and implementing an antenna. This lab is designed to reinforce concepts related to wave propagation. This work adds to the few papers in the literature reporting incorporation of innovative and low-cost experiments into the undergraduate electromagnetics curriculum (e.g. [1][4]).

At our university, the undergraduate electrical engineering curriculum contains two required electromagnetics classes. The first class focuses on electrostatic fields, and consists of lectures and tutorials. The second class focuses on timevarying fields, and has lectures, tutorials and laboratories. The first two labs include a series of multiple choice questions that allow students to practice problem solving related to time-varying fields and waves. These labs also incorporate simple hands-on experiments (e.g. dropping a magnet through a copper tube), as well as simulation tools (Amanogawa) to provide insight into wave propagation under varying conditions. Two additional labs use a simulation tool (Mefisto) to design transmission line circuits, providing students with insight into wave propagation characteristics on the lines. In 2017, we introduced a fifth lab to provide students with experience designing, implementing and testing an antenna. The students use the antenna to detect a "secret" radio transmission, and observe differences in reception under a variety of propagation conditions. This was a low-cost lab, as the purchase of materials for 80 students was less than
$\$ 1000$. Many of the components can be re-used in future years.

The antenna that the students implement is a Yagi-Uda antenna consisting of a driven element, a director and a reflector created with copper wire. Students are required to design the length and separation of the elements for operation at a specified frequency. We selected the 915 $\mathrm{MHz}$ industrial, scientific, medical (ISM) band, where lowpower signals can be legally transmitted. The antenna is assembled by attaching the elements to a wooden boom. The driven element is soldered to a coaxial line terminated with an SMA connector. To verify performance, students work with teaching assistants to measure the reflection from the antenna with a vector network analyzer (Keysight N9912A FieldFox). The element lengths are trimmed until desired performance is achieved.

The students connect their antennas to a software defined radio (RTL-SDR) and use the associated software (SDR Sharp) to demodulate a wideband FM-modulated signal transmitted from a radio source placed at the centre of the lab. By demodulating the received signal using the software, students hear the "secret code" transmitted by the radio source. Students then place a variety of objects between their receiving antenna and the transmitter, such as a metal plate, cardboard and RF absorbers, and comment on differences in the received signals. This exercise connects to class concepts such as wave propagation, transmission and reflection in different materials.

Students were surveyed specifically on this antenna lab through anonymous forms that requested student feedback about connection to concepts in the course, as well as the best part of the lab and suggestions for improvement. Most students commented that they enjoyed the hands-on experience of building an antenna. Students also commented on the connection of the lab to class concepts: "The lab was interesting in that it gave a new connection to wireless transmission and wave properties. The class is difficult in that it is not always intuitive to know exactly how a wave physically acts in space."; "For a course with so much concept and theories it is great to be able to apply 
what we have learned practically."; "It helped in understand wave propagation with regard to direction, as well as attenuation in varying material."; "A bit of a tangent. We don't talk about antennas much in this course. The experimentation with different materials, though, was more relevant."

In summary, this low-cost, hands-on lab provided students with an authentic learning experience that connected to the course materials. As electromagnetics classes are typically heavily focused on theory, this kind of lab has the potential to increase student engagement and depth of understanding.

\section{Acknowledgements}

We would like to thank the Department of Electrical and Computer Engineering for supporting the development of this lab, NSERC (PGS-M) and Alberta Innovates Technology Futures for funding, and the students and teaching assistants in ENEL 476 for their enthusiasm and feedback.

\section{References}

[1] J. Frolik, "Implementation of Handheld, RF Test Equipment in the Classroom and the Field," in IEEE Transactions on Education, vol. 50, no. 3, pp. 182-187, Aug. 2007. doi: 10.1109/TE.2007.900022

[2] S. V. Hum and M. Okoniewski, "A Low-Cost HandsOn Laboratory for an Undergraduate Microwave Course," in IEEE Antennas and Propagation Magazine, vol. 49, no. 3, pp. 175-184, June 2007.

[3] M. A. Jensen, D. V. Arnold and D. E. Crockett, "System-level microwave design: radar-based laboratory projects," in IEEE Transactions on Education, vol. 43, no. 4, pp. 414-419, Nov 2000. doi: $10.1109 / 13.883351$

[4] F. Torres, I. Corbella, A. Cardama, J. Romeu and O. Galindez, "Low cost transmission line demonstrator for a novel intermediate level communication engineering laboratory," in IEEE Transactions on Education, vol. 41, no. 4, pp. 13 pp.-, Nov 1998.Ed 